\title{
Radial variation of whistler-mode chorus: first results from the STAFF/DWP instrument on board the Double Star TC-1 spacecraft
}

\author{
O. Santolík ${ }^{1,2}$, E. Macúšová ${ }^{1}$, K. H. Yearby ${ }^{3}$, N. Cornilleau-Wehrlin ${ }^{4}$, and H. StC. K. Alleyne ${ }^{3}$ \\ ${ }^{1}$ Faculty of Mathematics and Physics, Charles University, V Holešovičkách 2, CZ-18000 Prague, Czech Republic \\ ${ }^{2}$ also at: IAP, Czech Academy of Sciences, Boční II/1401, CZ-14131 Prague, Czech Republic \\ ${ }^{3}$ University of Sheffield, Mappin Street, Sheffield, S1 3JD, UK \\ ${ }^{4}$ CETP/IPSL, 10-12 Av. de l'Europe, F-78140 Vélizy, France
}

Received: 22 February 2005 - Revised: 12 June 2005 - Accepted: 6 June 2005 - Published: 8 November 2005

Part of Special Issue "Double Star - First Results"

\begin{abstract}
We use the first measurements of the STAFF/DWP instrument on the Double Star TC-1 spacecraft to investigate whistler-mode chorus. We present initial results of a systematic study on radial variation of dawn chorus. The chorus events show an increased intensity at $L$ parameter above 6 . This is important for the possible explanation of intensifications of chorus, which were previously observed closer to the Earth at higher latitudes. Our results also indicate that the upper band of chorus at frequencies above one-half of the electron cyclotron frequency disappears for $L$ above 8 . The lower band of chorus is observed at frequencies below 0.4 of the electron cyclotron frequency up to $L$ of 11-12. The maxima of the chorus power spectra are found at slightly lower frequencies compared to previous studies. We do not observe any distinct evolution of the position of the chorus frequency band as a function of $L$. More data of the TC-1 spacecraft are needed to verify these initial results and to increase the MLT coverage.
\end{abstract}

Keywords. Magnetospheric physics (Plasma waves and instabilities) - Space plasma physics (Waves and instabilities) - Radio science (Magnetospheric physics)

\section{Introduction}

Whistler-mode chorus (Storey, 1953) is an electromagnetic wave emission occurring in the Earth's magnetosphere. It often contains many distinct short-duration wave packets which change their frequency at time scales of a fraction of a second (see reviews by Sazhin and Hayakawa, 1992; Omura et al., 1991). The generation of these wave packets is not yet well understood. It is most often accepted that chorus is

Correspondence to: O. Santolík

(ondrej.santolik@mff.cuni.cz) generated by a nonlinear process (Nunn et al., 1997; Trakhtengerts, 1999), based on the electron cyclotron resonance of whistler-mode waves with energetic electrons (Kennel and Petschek, 1966), taking place close to the geomagnetic equatorial plane (Burton and Holzer, 1974; LeDocq et al., 1998; Santolík et al., 2003; Parrot et al., 2003; Santolík et al., 2004, 2005). In its source region, chorus has been observed in two frequency bands (Burtis and Helliwell, 1976), separated by a (yet unexplained) narrow band of decreased intensity at onehalf of the local electron cyclotron frequency $\left(f_{\mathrm{ce}}\right)$. The importance of chorus is reflected by the recent discussion on the acceleration of energetic electrons in the outer radiation belt (e.g. Meredith et al., 2003).

The purpose of the present paper is to show that the STAFF/DWP instrument on board the Double Star TC-1 spacecraft (Cornilleau-Wehrlin et al., 2005; Yearby et al., 2005 ) is well suited for the investigation of chorus and to present the first results of a systematic study on radial variation of these emissions. The orbit of TC- 1 has a low inclination of $28.5^{\circ}$ with an apogee of 13.3 Earth radii $\left(R_{E}\right)$. The spacecraft thus stays at low geomagnetic latitudes during its orbit. It is then able to observe the chorus emissions close to their anticipated source region, and scan their variation as a function of the geocentric radial distance. For investigation of chorus, the TC- 1 orbit is complementary to the orbit of the Cluster spacefleet which crosses the chorus source region at a nearly constant radial distance of $4-5 R_{E}$ and in a roughly perpendicular direction with respect to the geomagnetic equator (Escoubet et al., 1997)

Chorus is known to frequently occur on the dawn side (see Sazhin and Hayakawa, 1992, and references therein). The orbits of the Double Star TC-1 spacecraft in its initial operational period were situated in this chorus-favorable sector. We could thus accumulate a number of observations, allowing us to investigate the radial evolution of chorus in a more 


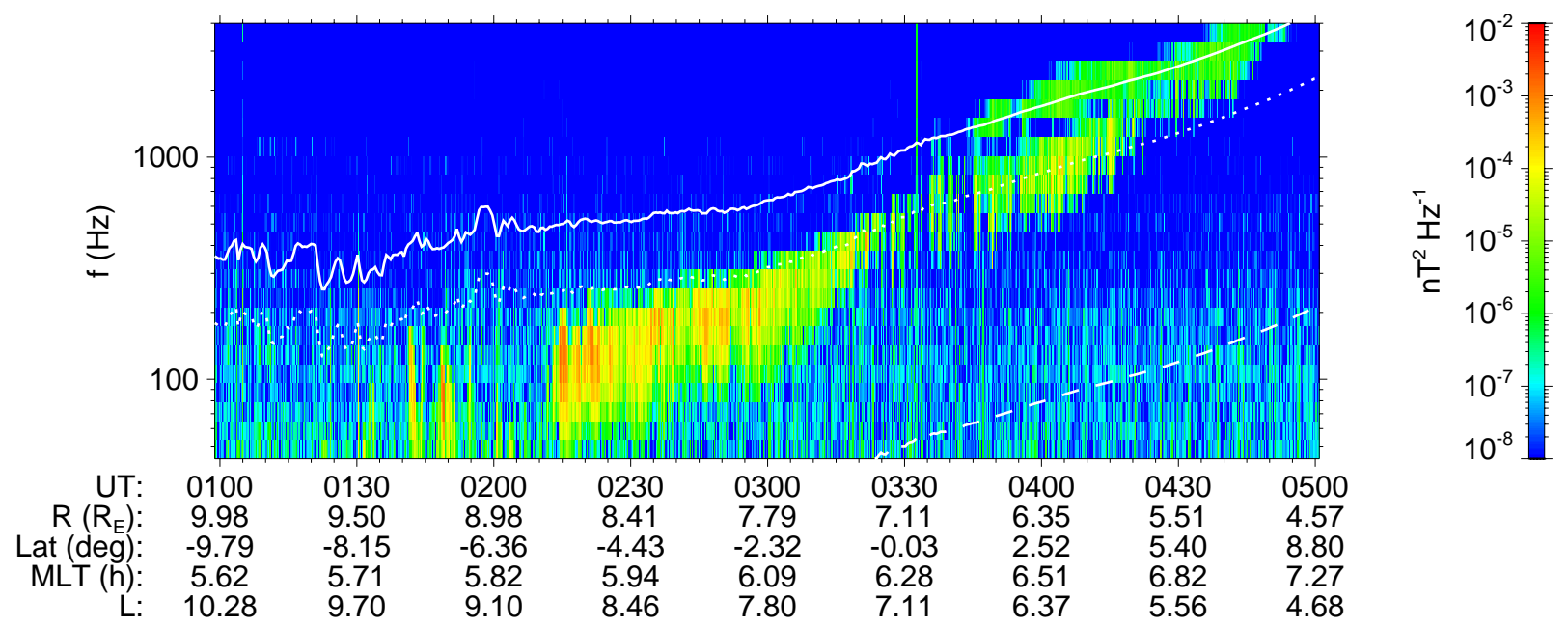

Fig. 1. Power-spectral density spectrogram of the magnetic field fluctuations measured during an inbound orbit on 28 June 2004 . The over-plotted white lines reflect different fractions of the local $f_{\text {ce }}$. Solid line: $f_{\text {ce }} / 2$; dotted line: $f_{\text {ce }} / 4$; dashed line: $f_{\text {ce }} / 42.85$ (approximation for the lower hybrid frequency). Spacecraft position is given on the bottom: R-geocentric radial distance; MLat-magnetic dipole latitude; MLT-magnetic local time; McIlwain's $L$ parameter.

systematic way. In spite of the failure of the STAFF antenna boom to deploy, we were able to estimate the power spectra of dawn chorus at different radial distances, ranging from the average plasmapause position up to the regions close to the magnetopause. This is very important since, as the ray tracing studies indicate (Parrot et al., 2004), chorus can propagate to higher latitudes from source locations distributed in such a wide range of radial distances. Using the initial measurements of the TC- 1 spacecraft, we address the important questions on the frequency band and relative intensity of chorus as a function of the geocentric radial distance.

In Sect. 2 we describe the data and show an example of chorus observations by the STAFF/DWP instrument on the TC-1 spacecraft. Section 3 presents our initial systematic analysis of the radial evolution of chorus; in Sect. 4 we discuss the obtained average properties of selected cases and, finally, in Sect. 5 we summarize these first results.

\section{Observations of chorus on board the TC-1 spacecraft}

The Double Star TC-1 spacecraft is equipped with a threeaxis search coil magnetometer STAFF which measures magnetic fluctuations at frequencies between $0.1 \mathrm{~Hz}$ and $4 \mathrm{kHz}$ (see Cornilleau-Wehrlin et al., 2005, for more details). The magnetometer signals are further processed by the Magnetic Wave Form (MWF) unit and by the Digital Wave Processor (DWP), which handles and formats the waveform data. At higher frequencies, the DWP unit also performs an onboard spectrum analysis, resulting in power spectra of the three components and their mutual phases in 27 frequency channels between $20 \mathrm{~Hz}$ and $4 \mathrm{kHz}$. The data set for the present paper comes from this onboard spectrum analysis, obtained with a time resolution of $1 \mathrm{~s}$. As discussed by CornilleauWehrlin et al. (2005), the STAFF magnetometer boom failed to deploy. As a consequence, the sensors are located close to the spacecraft body and the data are polluted by interference signals from the spacecraft systems. The received spectrumanalyzer data are therefore reprocessed using a noise reduction algorithm which includes a procedure to synthesize the power spectra in a direction where continuous interference is minimum (see Cornilleau-Wehrlin et al., 2005). This procedure has been used to process our entire data set. We further average the data to a 4-s time resolution which is sufficient for this study and provides us with a better signal-to-noise ratio.

Figure 1 shows an example of chorus observed by the TC1 spacecraft during an inbound orbit on 28 June 2004 between 01:00 and 05:00 UT. The spacecraft is located close to the magnetic equatorial plane during the entire time interval. Intense chorus emissions are observed starting at $\sim 02: 15$ UT and persist until the end of the interval, constantly shifting their frequency band to higher frequencies. For comparison, different fractions of the local $f_{\text {ce }}$ have been calculated using measurements of the onboard flux-gate magnetometer (Carr et al., 2005). At 02:15 UT, when the spacecraft is at large radial distances, chorus appears below one-quarter of the local $f_{\text {ce }}$. As the spacecraft moves closer to the Earth, around 04:00 UT, chorus becomes weaker and starts to occupy frequency bands above and below one-half of the local $f_{\text {ce. }}$. We can identify a narrow gap of decreased power just below $\frac{1}{2} f_{\text {ce }}$.

\section{Initial results of a systematic analysis of chorus power spectra}

A similar analysis has been performed for the data recorded between 13 January 2004 and 7 July 2004. We have visually inspected the results and selected a set of 25 continuous 
time intervals in 16 orbits where sufficiently intense chorus emissions have been identified. This limited set of data has been used in the subsequent analysis. It represents nearly $48 \mathrm{~h}$ of measurements containing approximately 42000 individual spectra. Figure 2a shows spacecraft positions during these time intervals in polar coordinates, where McIlwain's $L$ parameter is represented as the radius and the magnetic local time is plotted as the polar angle. The selected chorus events are located in the morning sector, at $L$ between 4 and 12.

Recent experimental studies based on Poynting flux and polarization measurements indicate that the dimension of the source region is a few thousands of $\mathrm{km}$ along the magnetic field lines (Santolík et al., 2003, 2004, 2005), and that its central position fluctuates around the geomagnetic equator. In Figure $2 b$ we present the selected data set with respect to a coordinate $s$ parallel to the magnetic field lines. This coordinate is defined as the arc length along the given field line measured from the geomagnetic equator, with the sign of the corresponding geomagnetic latitude. We have divided a larger interval of $s$ around the equator into 5000-km bins and the histogram in Fig. $2 b$ shows the observation time that the spacecraft spent during the selected events in each of these bins. The results indicate that most of these events have been recorded within several lengths of the source region from its expected position.

Each individual power spectrum obtained with a 4-s time resolution during these selected time intervals has been expressed as a function of the normalized frequency $f / f_{\text {ce }}$. Here, $f$ are the central frequencies of the 27 channels of the STAFF/DWF instrument. The local electron cyclotron frequency $f_{\text {ce }}$ is obtained for each spectrum from the onboard measurement of the ambient magnetic field, averaged again to the same 4-s time resolution. A value of the $L$ parameter has also been calculated for each spectrum from the actual spacecraft position.

The measured power-spectral densities have then been accumulated in the $L$-frequency bins, where we have used $32 L$ intervals between 3 and 11 , together with $25 f / f_{\text {ce }}$ intervals between 0.04 and 1.04. In each of these $32 \times 25$ bins we have calculated the mean value of the power-spectral density, based on the above described set of visually selected chorus events. We have calculated the mean values in separate bins using both arithmetic averages (in the linear scale) and geometric averages (in the logarithmic scale) of the accumulated power-spectral densities.

We have obtained qualitatively similar results from arithmetic and geometric averages and only the results for the arithmetic averages are shown in Fig. 3a, while Fig. 3b represents the number of power-spectral-density data points which were accumulated in each bin. We can see that the number of data points is highest at lower normalized frequencies. This is a consequence of the fact that the central frequencies of the 27 channels of the STAFF/DWF instrument are not evenly distributed on a linear scale. In fact, the channels were chosen to be displaced approximately evenly on a logarithmic frequency scale, i.e. the intervals between the

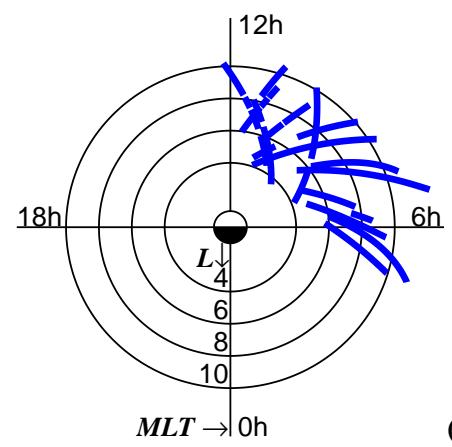

(a)

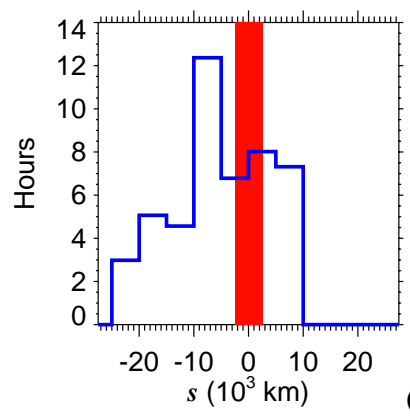

(b)

Fig. 2. Orbits of the TC-1 spacecraft during the selected chorus events. (a) Polar plot represents portions of orbits (thick blue lines) as a function of McIlwain's $L$ parameter and the magnetic local time (MLT). (b) Histogram shows number of hours spent by the spacecraft during the selected chorus events at different distances from the geomagnetic equator. The distances are calculated as arc lengths along the magnetic field lines; negative values of $s$ are used for observations at negative geomagnetic latitudes. The histogram bin size is $5000 \mathrm{~km}$. Red rectangle represents expected position of the chorus source region.

central frequencies are narrower at lower frequencies. Consequently, more data points fall into the lowest bins of our linear scale of normalized frequencies. The white area on the top left is again an artifact connected to the upper frequency limit of the instrument $(4 \mathrm{kHz})$, which is below the local $f_{\text {ce }}$ when the spacecraft approaches the perigee at lower $L$ values. The large numbers of data points at $L$ between 6 and 11 reflect the distribution of the number of selected chorus cases as a function of $L$. Figure $3 \mathrm{~b}$ shows that, except for the bins where the instrument does not measure at all (white area), the numbers of power-spectral density points in separate bins range from a few hundred to a few thousand. These high numbers indicate that the mean values shown in Fig. 3a have a very good statistical significance.

The resulting average power-spectral densities in Fig. 3 are largest for higher $L$ (above $\sim 6-7$ ). Intense chorus here generally occurs at normalized frequencies below $f / f_{\mathrm{ce}}=0.4$, with no clear dependence of this upper limit on $L$. The maximum power-spectral densities are then observed for $f / f_{\text {ce }}$ between 0.1 and 0.3 . At lower $L$ (below $\sim 8$ but mainly around $L \sim 5$ ), a signature of the upper band of chorus appears above $f / f_{\text {ce }}=0.5$. At $L \gtrsim 8$ this signature disappears and only the lower-band chorus remains. 


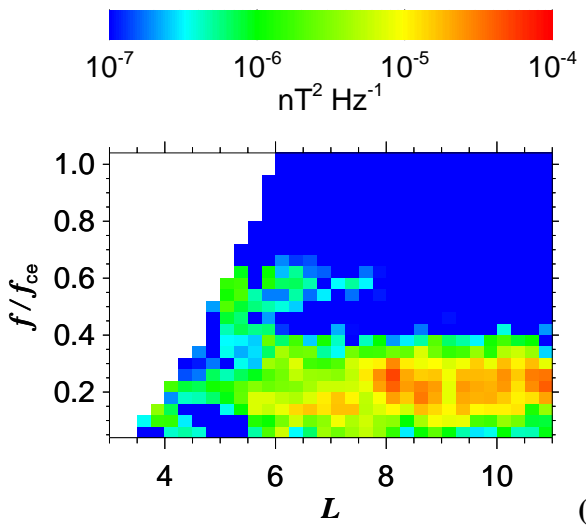

(a)

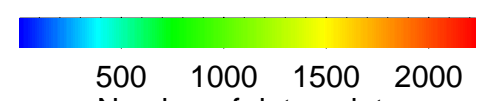

Number of data points

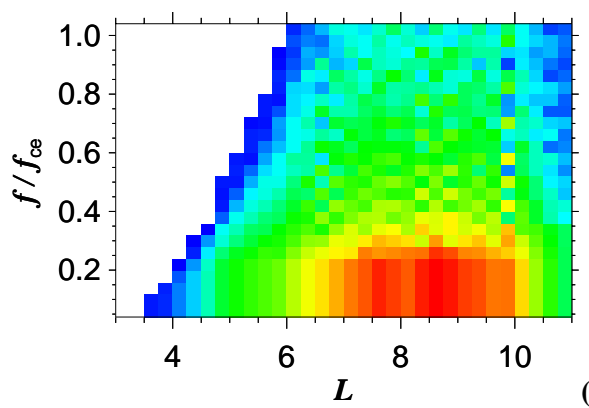

(b)

Fig. 3. (a) Average power-spectral density spectrogram as a function of McIlwain's $L$ parameter and the wave frequency $f$ normalized by the local $f_{\text {ce }} .42000$ individual spectra recorded during the orbits from Fig. 2 have been accumulated. (b) Number of powerspectral density points used in each bin of Fig. 3a.

To verify the characteristic chorus frequencies, we have further selected a subset of spectra which were recorded at $L \geq 6$ and which have their maxima above $10^{-4} \mathrm{nT}^{2} \mathrm{~Hz}^{-1}$. In this subset of 4300 spectra, we have determined normalized frequencies where the individual spectra have their maxima. A histogram of these characteristic normalized frequencies is shown in Fig. 4. The result indicates a bimodal distribution with peaks at $f / f_{\text {ce }}$ of $\sim 0.15$ and $\sim 0.24$. The main distribution of these characteristic chorus frequencies is contained in an interval between 0.05 and $0.35 f_{\text {ce }}$.

\section{Discussion}

The histogram in Fig. 4, showing a bimodal feature, should be taken as a very preliminary result. The data from only 16 orbits which are included in this study have a very limited spatial coverage, and detailed features on the histogram might be strongly influenced by this limitation. A more comprehensive study based on a larger data set is needed, and only a rough shape of the distribution of characteristic frequencies should be considered as a result of the present study.

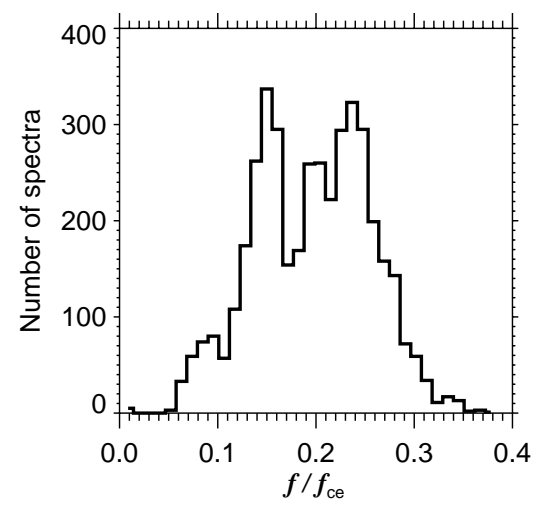

Fig. 4. Histogram of normalized frequencies of maximum powerspectral density for intense chorus events from Fig. 2 occurring at $L>6$.

However, the histogram in Fig. 4 still gives lower characteristic frequencies compared to the results of the statistical study of Burtis and Helliwell (1976), who, using data measured in 1966-1967 by the OGO 3 spacecraft, found the peak of the distribution at a frequency close to $0.35 f_{\text {ce }}$. Several possible reasons for this slight discrepancy can be found. First, our method to estimate the characteristic frequency is different from the method of Burtis and Helliwell (1976), who analyzed separate chorus elements. Here we use only a rough procedure based on the maxima of averaged power spectra. This could induce a systematic difference in results. More importantly, we use the local $f_{\text {ce }}$ to normalize the wave frequency. As the spacecraft orbit is not located exactly at the geomagnetic equator, the local $f_{\text {ce }}$ is always slightly higher than $f_{\text {ce }}$ which corresponds to the equatorial crossing of the same field line. This effect would move the peak of normalized frequencies to lower values. If it is confirmed by future detailed studies, it may, at least partially, explain the observed discrepancy. If the difference cannot be explained by these most likely reasons, it could also be indicative of a long-term variation in the statistical behavior of chorus waves, possibly related to the long-term variations in the magnetosphere connected with the particular phase of the solar cycle. A further analysis of this difference might thus be useful.

Another relevant past study is that of Koons and Roeder (1990), who analyzed broad-band VLF data of the SCATHA satellite during one year of operation in 1979-1980. They found chorus during more than $70 \%$ of the observation time in the dawn-noon sector at $L=5-8$. They also analyzed the occurrence rate of the chorus emissions that displayed a frequency gap at $\frac{1}{2} f_{\text {ce. They found that these emissions }}$ generally followed the same spatial distribution as all chorus but with a reduced occurrence rate. The chorus without the gap was not associated with any upper-band emissions above $\frac{1}{2} f_{\text {ce }}$. The limited data set in our present study does not allow us to directly compare the results on occurrence rates. However, our Fig. 3a shows that the upper band emissions disappear from the average spectrogram of selected chorus 
cases at $L \gtrsim 8$. This might still be consistent with the results of Koons and Roeder (1990), since their analysis is done below $L<8$. The absence (or strong attenuation) of chorus at frequencies above $\frac{1}{2} f_{\text {ce }}$ at $L$ above $\sim 8$ thus needs to be verified by further studies.

Concerning the lower-band chorus below $\frac{1}{2} f_{\text {ce }}$, Fig. 3a shows that the average power spectrogram of selected chorus events shows a higher intensity at higher $L$ above $\sim 6$. Using the results of ray-tracing studies (e.g. Parrot et al., 2004), this region can contain sources of chorus which subsequently propagate to higher latitudes and toward the Earth. These waves can be observed by polar orbiting spacecraft at lower radial distances, such as Cluster. If a further study based on a larger data set confirms these results, it could be possible to explain the intensifications of chorus at higher latitudes (e.g. Meredith et al., 2001) just by having more intense chorus generated close to the magnetic equator at higher $L$.

In Fig. 3a, the lower band of chorus does not show any clear variation of its characteristic frequency band as a function of $L$ in the interval from $L \sim 5$ up to $L \sim 11-12$. This, together with the observed absence of the upper-band chorus at $L \gtrsim 8$, might be an important test for existing nonlinear theories on chorus generation (e.g. Trakhtengerts, 1999).

\section{Conclusions}

We have demonstrated that the measurements of the STAFF/DWP instrument on board the Double Star TC-1 spacecraft are a good tool for the investigation of whistlermode chorus. We have presented the initial results of a systematic study on the radial variation of dawn chorus emissions. The TC-1 orbit is especially favorable for this investigation since the spacecraft scans a large range of geocentric radial distances, while staying close to the magnetic equatorial plane where the source region of chorus is expected.

The most important result is a higher intensity of chorus at $L$ above $\sim 6$, shown on the average power spectrogram obtained from selected chorus events. The lower band of chorus below $\frac{1}{2} f_{\text {ce }}$ is observed to be contained at frequencies below $0.4 f_{\text {ce }}$ up to $L$ of $11-12$. The maximum power-spectral densities are found in an interval between 0.05 and $0.3 f_{\text {ce }}$, i.e. at slightly lower frequencies compared to previous statistical studies. No clear evolution of the position of this band is observed as a function of $L$. The signature of the upper band of chorus above $\frac{1}{2} f_{\text {ce }}$ is absent in the average spectrogram of selected chorus cases at $L \gtrsim 8$.

Further chorus studies based on more data of the TC-1 spacecraft are needed to verify these first results. The MLT coverage presented here was limited to the morning sector. Future observations on the nightside will be especially interesting, because of the importance of the nightside storm-time chorus for the dynamics of energetic electrons in the outer radiation belt. Simultaneous measurements of the Cluster spacefleet and the TC-1 spacecraft will also be very interesting since they open the possibility to observe the same chorus emissions in their source region at higher $L$ by TC-1 and also by Cluster, after they propagated to higher latitudes toward the Earth.

Acknowledgements. The Double Star TC-1 STAFF/DWP experiment was funded by ESA. We thank C. Carr and the FGM team for the use of the flux-gate magnetometer data. This work was supported by the ESA PECS contract No. 98025 and by grants GACR202/03/0832 and 1P05ME811.

Topical Editor T. Pulkkinen thanks U. S. Inan and another referee for their help in evaluating this paper.

\section{References}

Burtis, W. J. and Helliwell, R. A.: Magnetospheric chorus: Occurrence patterens and normalized frequency, Planet. Space Sci., 24, 1007-1024, 1976.

Burton, R. K. and Holzer, R. E.: The origin and propagation of chorus in the outer magnetosphere, J. Geophys. Res., 79, 10141023, 1974.

Carr, C. M., Brown, P., Zhang, T. L., et al.: The Double Star magnetic field investigation: overview of instrument performance and initial results, Ann. Geophys., 23, 2713-2732, 2005.

Cornilleau-Wehrlin, N., Alleyne, H., Yearby, K. H., et al.: The STAFF-DWP wave instrument on the DSP equatorial spacecraft: description and first results, Ann. Geophys., 23, 2785-2801, 2005.

Escoubet, C. P., Schmidt, R., and Goldstein, M. L.: Cluster - Science and Mission Overview, Space Science Reviews, 79, 11-32, 1997.

Kennel, C. F. and Petschek, H. E.: Limit on stable trapped particle fluxes, J. Geophys. Res., 71, 1-28, 1966.

Koons, H. C. and Roeder, J. L.: A survey of equatorial magnetospheric wave activity between 5 and $8 \mathrm{R}_{E}$, Planet. Space. Sci., 38, 1335-1341, 1990.

LeDocq, M. J., Gurnett, D. A., and Hospodarsky, G. B.: Chorus source locations from VLF Poynting flux measurements with the Polar spacecraft, Geophys. Res. Lett., 25, 4063-4066, 1998.

Meredith, N. P., Horne, R. B., and Anderson, R. R.: Substorm dependence of chorus amplitudes: Implications for the acceleration of electrons to relativistic energies, J. Geophys. Res., 106, 13 165-13 178, 2001.

Meredith, N. P., Cain, M., Horne, R. B., Thorne, R. M., Summers, D., and Anderson, R. R.: Evidence for chorus-driven electron acceleration to relativistic energies from a survey of geomagnetically disturbed periods, J. Geophys. Res., 108(A6), 1248, doi:10.1029/JA009764, 2003.

Nunn, D., Omura, Y., Matsumoto, H., Nagano, I., and Yagitani, S.: The numerical simulation of VLF chorus and discrete emissions observed on the Geotail satellite using a Vlasov code, J. Geophys. Res., 102, 27 083-27 097, 1997.

Omura, Y., Nunn, D., Matsumoto, H., and Rycroft, M. J.: A review of observational, theoretical and numerical studies of VLF triggered emissions, J. Atmos. and Terr. Phys., 53, 351-368, 1991.

Parrot, M., Santolík, O., Cornilleau-Wehrlin, N., Maksimovic, M., and Harvey, C.: Source location of chorus emissions observed by Cluster, Ann. Geophys., 21, 473-480, 2003,

SRef-ID: 1432-0576/ag/2003-21-473.

Parrot, M., Santolík, O., Gurnett, D., Pickett, J., and CornilleauWehrlin, N.: Characteristics of magnetospherically reflected chorus waves observed by CLUSTER, Ann. Geophys., 22, 2597- 
2606, 2004,

SRef-ID: 1432-0576/ag/2004-22-2597.

Santolík, O., Gurnett, D. A., Pickett, J. S., Parrot, M., and Cornilleau-Wehrlin, N.: Spatio-temporal structure of storm-time chorus, J. Geophys. Res., 108, 2003.

Santolík, O., Gurnett, D. A., Pickett, J. S., Parrot, M., and Cornilleau-Wehrlin, N.: A microscopic and nanoscopic view of storm-time chorus on 31 March 2001, Geophys. Res. Lett., 31(2), L02801, doi:10.1029/2003GL018757, 2004.

Santolík, O., Gurnett, D. A., Pickett, J. S., Parrot, M., and Cornilleau-Wehrlin, N.: Central position of the source region of storm-time chorus, Planet. Space Sci., 53, 299-305, 2005.
Sazhin, S. S. and Hayakawa, M.: Magnetospheric chorus emissions: A review, Planet. Space Sci., 40, 681-697, 1992.

Storey, L. R. O.: An investigation of whistling atmospherics, Phil. Trans. Roy. Soc. London, A246, 113-141, 1953.

Trakhtengerts, V.: A generation mechanism for chorus emission, Ann. Geophys., 17, 95-100, 1999,

SRef-ID: 1432-0576/ag/1999-17-95.

Yearby, K. H., Alleyne, H., Cornilleau-Wehrlin, N., et al.: Observations of lion roars in the magnetosheath by the STAFF/DWP experiment on the Double Star TC-1 spacecraft, Ann. Geophys., 23, 2861-2866, 2005. 